\title{
Leaky Mode Resonance-Induced Sensitive Ultraviolet Photodetector Composed of Graphene/Small Diameter Silicon Nanowire Array Heterojunctions
}

Jun-Jie Wang, ${ }^{\dagger}$ Can Fu, ${ }^{\dagger}$ Hai-Yang Cheng, ${ }^{\dagger}$ Xiao-Wei Tong, ${ }^{\dagger}$ Zhi-Xiang Zhang, ${ }^{\dagger}$ Di Wu, ${ }^{*}$ Li-Miao Chen," Feng-Xia Liang, ${ }^{\S} *$ Lin-Bao Luo ${ }^{\dagger} *$

† School of Electronic Science and Applied Physics, Hefei University of Technology, Hefei, 230009, China

$\mp$ Key Laboratory of Materials Physics of Ministry of Education, Department of Physics and Engineering, Zhengzhou University, Zhengzhou 450052, China

§ School of Materials Science and Engineering and Anhui Provincial Key Laboratory of Advanced Functional Materials and Devices, Hefei University of Technology, Hefei 230009, China

" Hunan Provincial Key Laboratory of Micro \& Nano Materials Interface Science, College of Chemistry and Chemical Engineering, Central South University, Changsha 410083, China

* Corresponding authors. E-mail: fxliang@hfut.edu.cn (F. X. Liang), luolb@hfut.edu.cn (L. B. Luo) 

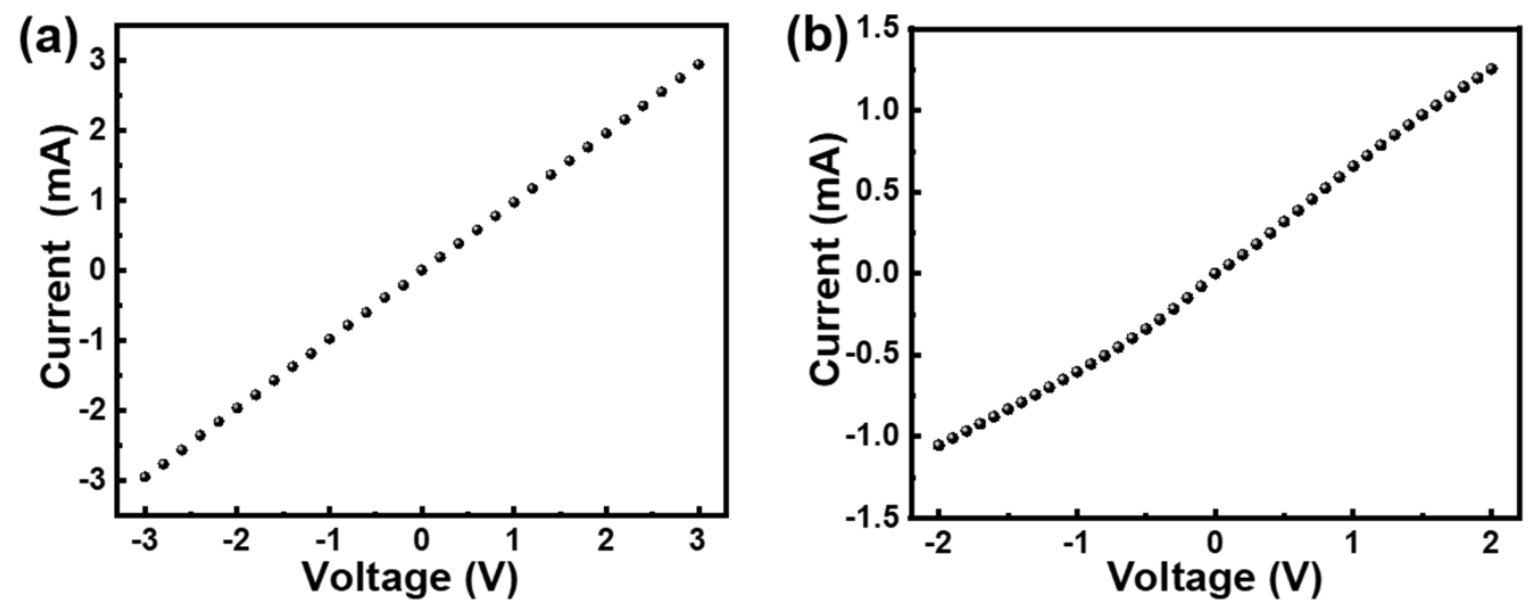

Figure S1. (a) $I-V$ curve of the $\mathrm{Ag} / \mathrm{Graphene} / \mathrm{Ag}$ contact. (b) $I-V$ curve of the $\mathrm{In}-\mathrm{Ga} / \mathrm{n}-\mathrm{Si} / \mathrm{In}-\mathrm{Ga}$ contact.

(a)

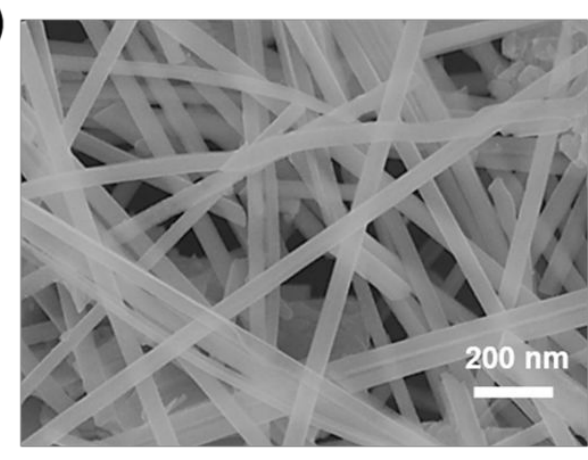

(b)

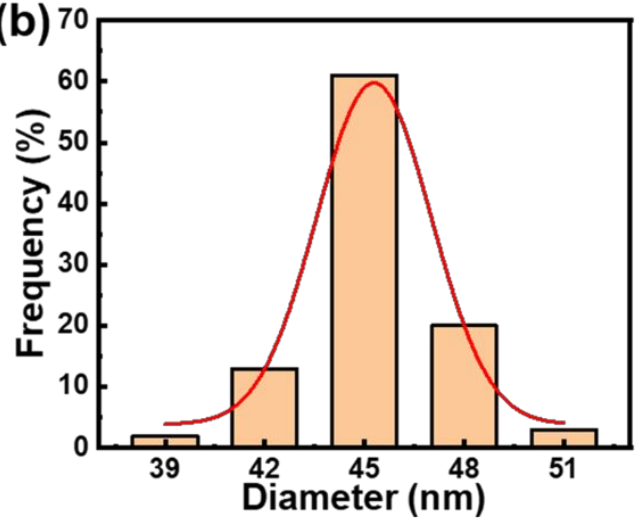

Figure S2. (a) SEM images of scraped SiNWs. (b) Diameter distribution of SiNWs. 


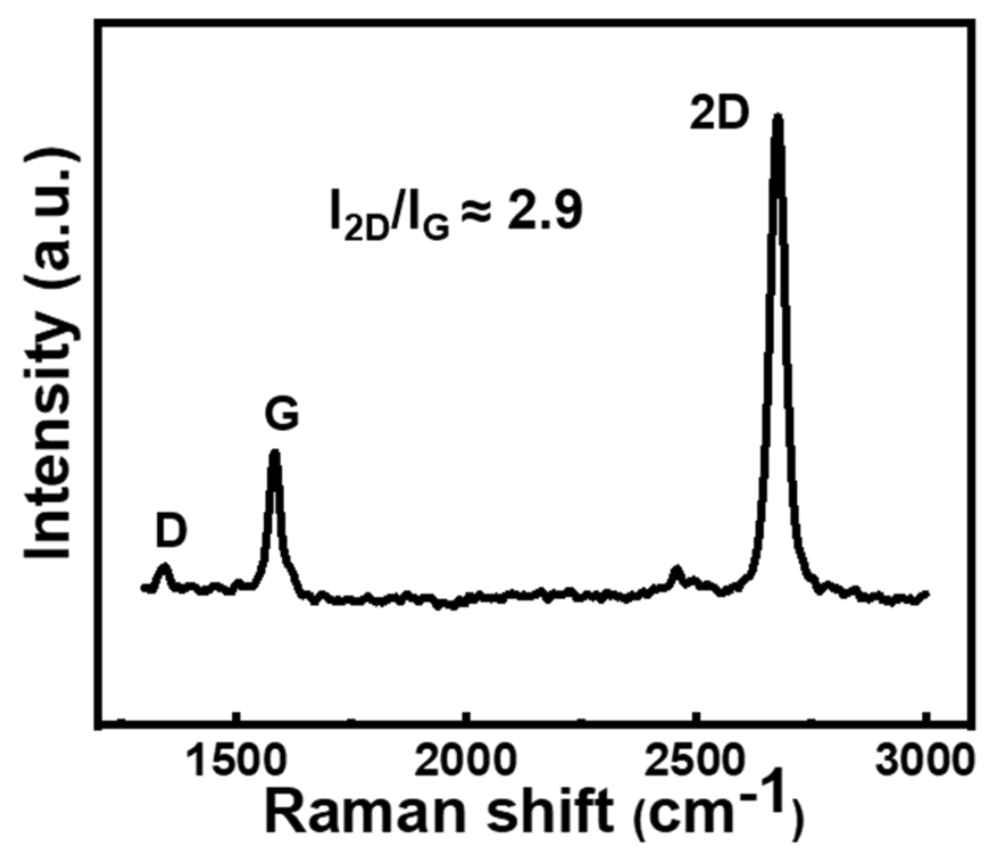

Figure S3. Raman spectrum of the graphene film.

\section{Solution of leaky modes:}

By solving the dispersion relation, the leaky modes in the cylinder with radius $\mathrm{R}$ are obtained:1,2

$\left[\frac{\mu_{c} J_{m}^{\prime}\left(k_{c} R\right)}{k_{c} R J_{m}\left(k_{c} R\right)}-\frac{\mu H_{m}^{\prime}(k R)}{k R H_{m}(k R)}\right]\left[\frac{\varepsilon_{c} J_{m}^{\prime}\left(k_{c} R\right)}{k_{c} R J_{m}\left(k_{c} R\right)}-\frac{\varepsilon H_{m}^{\prime}(k R)}{k R H_{m}(k R)}\right]=m^{2} \frac{\left(k_{z} R\right)^{2}}{(\omega R / c)^{2}}\left(\frac{1}{(k R)^{2}}-\frac{1}{\left(k_{c} R\right)^{2}}\right)^{2}$

where $m$ is an integer, and $J_{m}$ and $H_{m}$ are standard Bessel and (first-kind) Hankel functions. The mode wavevectors inside $\left(k_{c}\right)$ and outside $(k)$ the cylinder at frequency $\omega$ are the transverse components of the complex wavevector $\left(k_{z}\right)$ along the cylinder axis in vacuum. The dielectric permittivities and magnetic permeabilities of the cylinder and the medium are $\varepsilon_{c}, \mu_{c}$ and $\varepsilon, \mu$, respectively, with corresponding refractive indices $n_{c}=\left(\varepsilon_{c} \mu_{c}\right)^{1 / 2}$ and $n=(\varepsilon \mu)^{1 / 2}$.

Leaky modes are classified according to their azimuthal mode number (m), radial mode number (n), and their polarization. Their polarization can be TM (transverse magnetic, $\mathrm{H}_{Z}=0$ ), TE (transverse electric, $\left.E_{Z}=0\right)$, HE (mag-netoelectric, TM-like), or EH (electromagnetic, TE-like). 
For arbitrary wave vectors, the only mode that is strictly TE or TM is the 0 -order azimuth mode. ${ }^{3}$ In the case of $m=0$, the solution of equation (1) produces two kinds of equations:

$$
\begin{array}{ll}
\frac{\varepsilon_{c} J_{m}^{\prime}\left(k_{c} R\right)}{k_{c} R J_{m}\left(k_{c} R\right)}-\frac{1 H_{m}^{\prime}(k R)}{k R H_{m}(k R)}=0 & \text { for TE modes } \\
\frac{1 J_{m}^{\prime}\left(k_{c} R\right)}{k_{c} R J_{m}\left(k_{c} R\right)}-\frac{1{H_{m}^{\prime}}^{\prime}(k R)}{k R H_{m}(k R)}=0 & \text { for TM modes }
\end{array}
$$

When $\mathrm{m}>0$ or the electromagnetic wave is parallel to the nanowire axis, only the $\mathrm{HE}_{1 \mathrm{~m}}$ leakage mode causes the absorption enhancement due to symmetry matching requirements. ${ }^{4,5}$

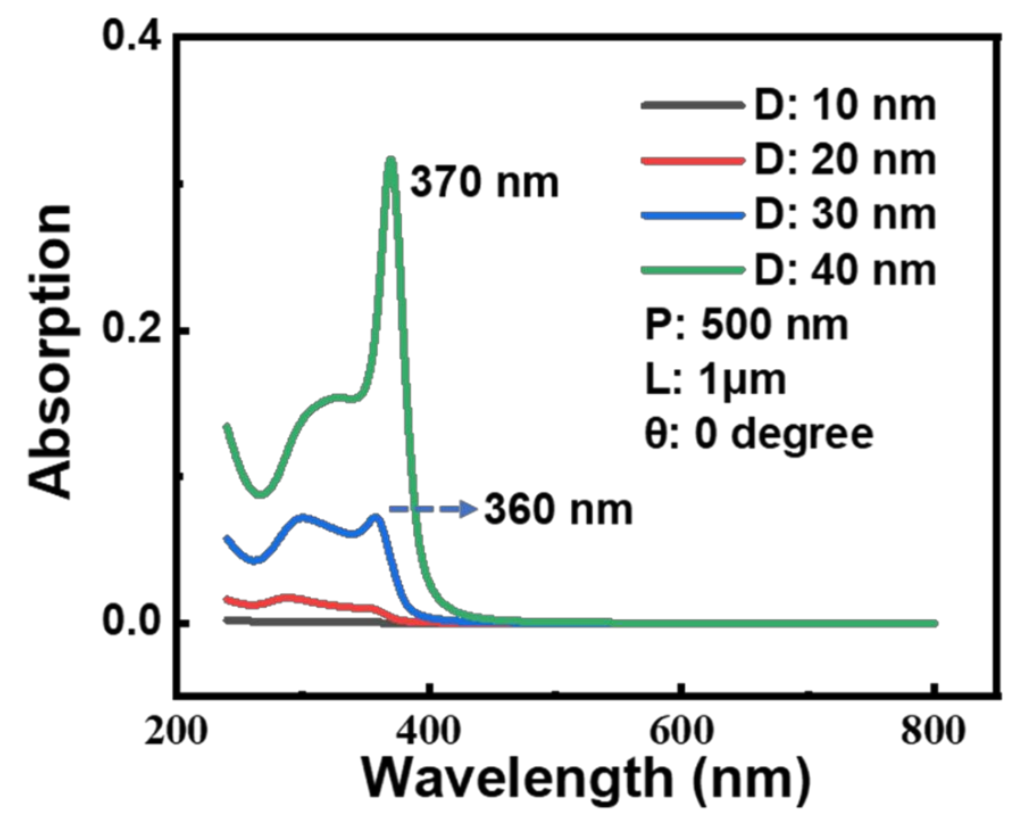

Figure S4. The absorption curve of SiNWs array with different diameters (10, 20, 30, and $40 \mathrm{~nm})$. 

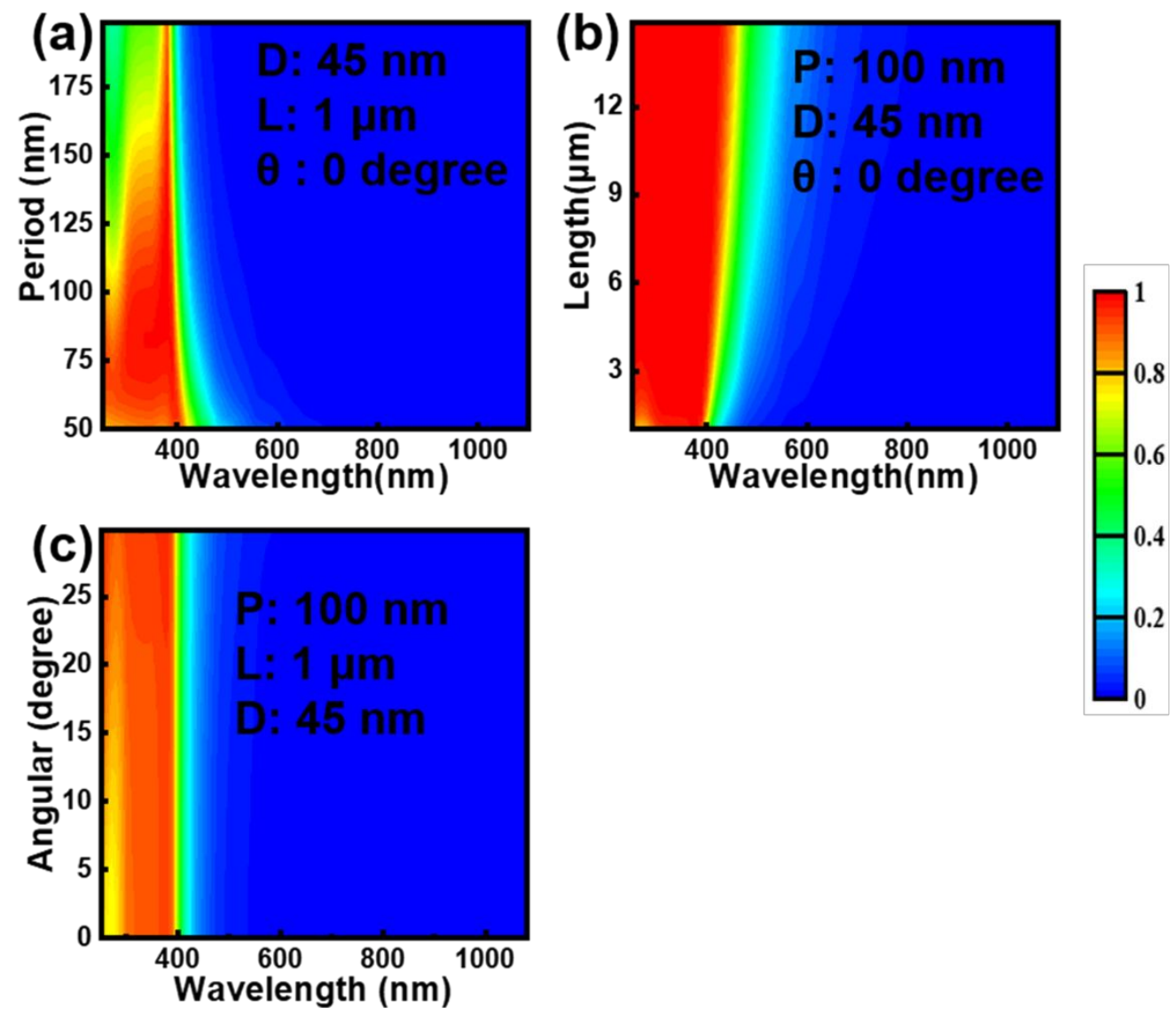

Figure S5. Contour plot of the absorption of vertical SiNWs array as a function of wavelength and (a) period, (b) length, and (c) angular, respectively.
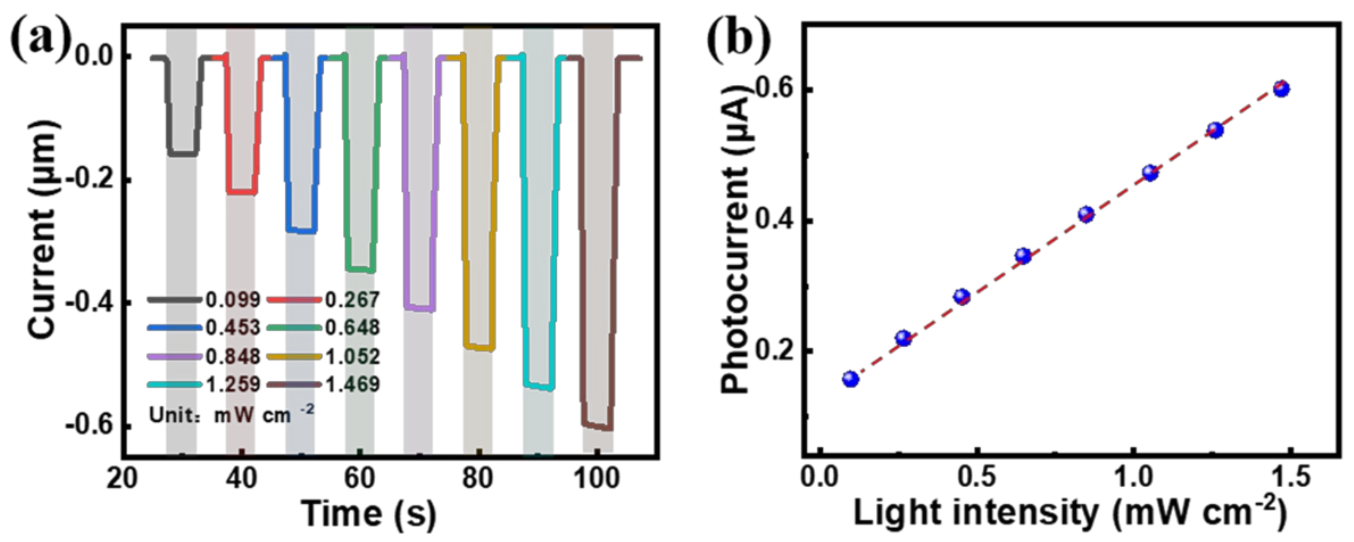

Figure S6. (a) photoresponse curves of the MLG/SiNWs array heterojunction UVPD under $365 \mathrm{~nm}$ light with different intensities. (b) The photocurrent is a linear function of the incident light intensity. 


\section{Solution of key parameters of the photodetector:}

The responsivity $(\mathrm{R})$ is photocurrent per unit of incident light power in the effective illumination area, which can be evaluated with the following formula:

$R=\frac{I_{\lambda}-I_{d}}{P_{\lambda} S}$

Where $I_{\lambda}$ is the photocurrent, $P_{\lambda}$ is the light intensity $\left(P_{\lambda}=0.5 \mathrm{~mW} \mathrm{~cm}{ }^{-2}\right), I_{d}$ is the dark current $\left(\mathrm{I}_{\mathrm{d}}=-2\right.$

$\left.\times 10^{-9} \mathrm{~A}\right)$, and $S$ is the effective illuminated area $\left(\mathrm{S} \approx 4 \times 10^{-3} \mathrm{~cm}^{2}\right)$.

$E Q E$ is defined as the number of electrons detected per incident photon, which can be calculated as follows:

$E Q E=\frac{h c R_{\lambda}}{(e \lambda)}$

where $h$ is the Planck constant $\left(6.626 \times 10^{-34} \mathrm{~J} \cdot \mathrm{s}\right), c$ is the speed of light $\left(3 \times 10^{8} \mathrm{~m} / \mathrm{s}\right), e$ is the electron charge $\left(1.602 \times 10^{-19} \mathrm{C}\right)$, and $\lambda$ is the excitation wavelength. The $D *$ is calculated to evaluate its ability to detect the smallest optical signals:

$D *=\frac{R \cdot S^{1 / 2}}{\left(2 e \cdot I_{d}\right)^{1 / 2}}$

Where e is the electronic charge $\left(1.602 \times 10^{-19} \mathrm{C}\right)$. 


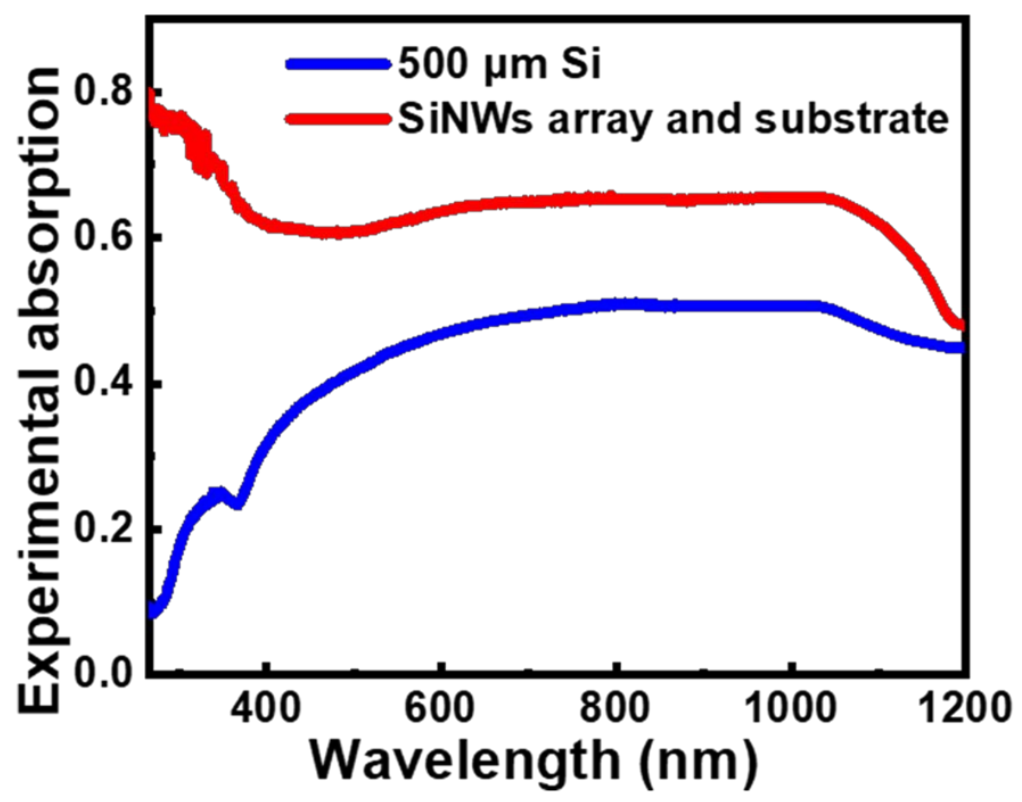

Figure S7. Experimental absorption spectra of both SiNWs array etched from Si substrate and a planar $500 \mu \mathrm{m}$ thick Si substrate.

Solution of the width of depletion region:

The following formula obtains the ideal width of depletion layer:

$$
W_{\text {dep }}=\sqrt{2 \varepsilon_{s}\left(\Psi_{b i}-V-k_{0} T / q\right) / q N_{d}}
$$

Where $\varepsilon_{s}, \Psi_{b i}, N_{d}$, and q are dielectric constant $\left(\mathrm{Si}, \varepsilon_{s}=1.053 \times 10^{-12}\right)$, built-in potential $\left(\Psi_{b i}=W_{G r}\right.$ $\left.W_{S i}=0.29 \mathrm{eV}\right)$, the donor impurity concentration of semiconductor $\left(\sim 10^{15}\right)$ and the electron charge $\left(1.602 \times 10^{-19} \mathrm{C}\right)$, respectively. The $k_{0} T / q$ at room temperature is usually ignored. ${ }^{6} W_{d e p}$ is calculated to be $\sim 0.6 \mu \mathrm{m}$ at 0 bias. 


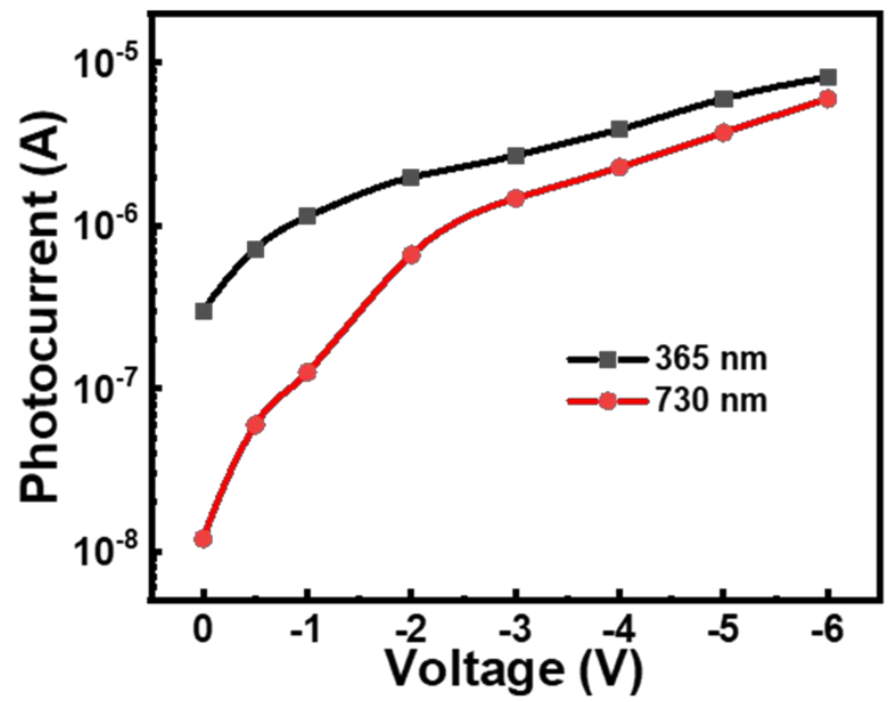

Figure S8. The photocurrent generated by the incident light at $365 \mathrm{~nm}$ and $730 \mathrm{~nm}$ as a function of bias voltage, the power intensities are all set to be $0.5 \mathrm{~mW} \mathrm{~cm}^{-2}$.

\section{Reference}

(1) Abujetas, D. R.; Paniagua-Domínguez, R.; Sánchez-Gil, J. A. Unraveling the Janus Role of Mie Resonances and Leaky/Guided Modes in Semiconductor Nanowire Absorption for Enhanced Light Harvesting. ACS Photonics 2015, 2, 921-929.

(2) Stratton, J. A. Electromagnetic Theory, 1st ed.; McGraw-Hill, Inc.: New York, 1941.

(3) Fountaine, K. T.; Whitney, W. S.; Atwater, H. A. Resonant Absorption in Semiconductor Nanowires and Nanowire Arrays: Relating Leaky Waveguide Modes to Bloch Photonic Crystal Modes. J. Appl. Phys. 2014, 116, 153106.

(4) Wang, B.; Leu, P. W. Tunable and Selective Resonant Absorption in Vertical Nanowires. Opt. Lett. 2012, 37, 3756-3758.

(5) Snyder, A. W.; Love, J. Optical Waveguide Theory; Springer US: Boston, MA, 1983; pp 238-279.

(6) Sze, S. M. Physics of Semiconductor Devices; Wiley: New York, 1981. 